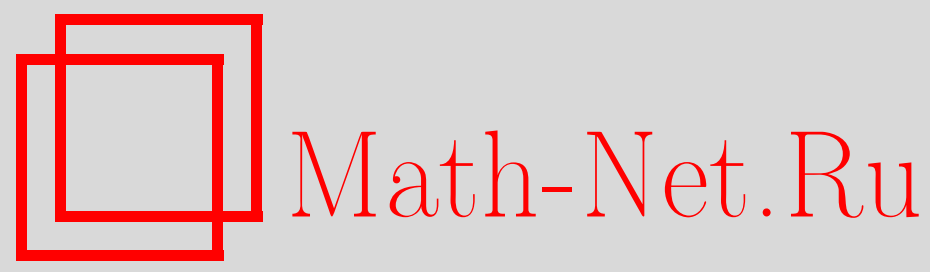

Б. П. Куфарев, Н. Г. Никулина, Об интегрировании по уровням функций класса ACL, Матем. заметки, 2005, том 77 , выпуск 4, 540-543

DOI: https://doi.org/10.4213/mzm2515

Использование Общероссийского математического портала Math-Net.Ru подразумевает, что вы прочитали и согласны с пользовательским соглашением http://www . mathnet.ru/rus/agreement

Параметры загрузки:

IP: 3.91 .87 .62

26 апреля 2023 г., 09:06:51

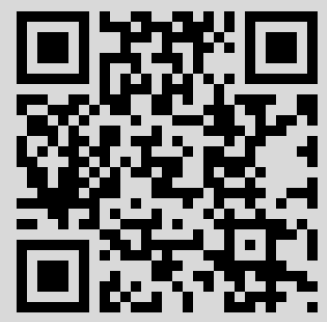




\title{
ОБ ИНТЕГРИРОВАНИИ ПО УРОВНЯМ ФУНКЦИЙ КЛАССА АСL
}

\section{Б. П. Куфарев, Н. Г. Никулина}

\begin{abstract}
Классическая формула повторного интегрирования распространяется на множества уровня ACL-функций.

Библиографиия: 9 названий.
\end{abstract}

Обозначим через $\mathrm{ACL}_{p}$ множество всех непрерывных в области $G \subset \mathbb{R}^{n}$ функций $r(x)$ класса $L_{p, \text { lос }}^{1}$ в области $G$. Пусть $H$-хаусдорфова $(n-1)$-мера и $\underset{t}{E}=\{x \in E \mid r(x)=t\}$ при $E \subset G$.

Для абсолютно непрерывных (по Кронроду [1]) на замкнутом прямоугольном параллелепипеде (брусе) $I \subset G$ функций $r(x)$ формула

$$
\int_{I}|\nabla r(x)| d x=\int_{r(I)} H(\underset{t}{I}) d t=: V(r, I)
$$

доказана еще в начале 50-х годов российскими математиками, см. [1], [2]. Потом она появилась у де Джорджи [3] в предположении, что $r(x)$ дифференцируема, и была обобщена Г. Федерером [4] на случай липшицевых отображений $r(x)$ многообразия размерности $n$ в $m$-мерное многообразие, $n \geqslant m$. О дальнейшем распространении ее см. [5].

ЛЕмма. Формула (1) верна для $r(x) \in \mathrm{ACL}_{1}(G)$.

ДокАЗАТЕЛЬСтво. Так как функция $r(x)$ принадлежит соболевскому классу $W_{1, \text { loc }}^{1}(G)$, существует, как известно, последовательность функций $r_{l} \in C^{\infty}(G)$ такая, что $r_{l}$ равномерно сходятся к $r$ на каждом компакте $K \subset G$ и $\nabla r_{l} \rightarrow \nabla r$ в $L_{1}(K)$ при $l \rightarrow \infty, l=1,2, \ldots$. Но для $r_{l}$ на любом замкнутом брусе $I \subset G$ вариация

$$
V\left(r_{l}, I\right)=\int_{I}\left|\nabla r_{l}\right| d x
$$

см. (1), и так как $n$-мерная вариация $V(r, I)$ является функционалом, полунепрерьвным снизу относительно равномерной сходимости (см. [2, 19 и $§ 22])$, то

$$
V(r, I) \leqslant \frac{\lim }{l} V\left(r_{l}, I\right)=\lim _{l} \int_{I}\left|\nabla r_{l}\right| d x=\int_{I}|\nabla r| d x
$$

т.е. $n$-мерная вариация функции $r$ ограничена на каждом замкнутом брусе $I \subset G$. 
Докажем, что из $(*)$ следует абсолютная непрерывность вариации $V(r, A)$ как функции множества $A \subset B$, где $B$ - произвольная фиксированная ограниченная область с замьканием $\bar{B} \subset G$. Действительно, $\forall \varepsilon>0$ существует такое $\delta=\delta(\varepsilon)$, что если $e \subset B$ и $\operatorname{mes}_{n} e<\delta$, то

$$
\int_{e}|\nabla r| d x<\varepsilon
$$

Пусть $A \subset B$ - ограниченное борелевское множество с мерой меньше $\delta$. Покроем $A$ счетной системой входящих в $B$ и взаимно неналегающих полуоткрытых брусов $\left\{I_{j}\right\}$, $j=1,2, \ldots$, с суммой объемов меньше $\delta$ (грани брусов параллельны координатным плоскостям). Тогда в силу полной адлитивности $n$-мерной вариации на классе борелевских множеств и в силу $(*)$

$$
V(r, A) \leqslant V\left(r, \bigcup_{I} I_{j}\right)=\sum_{j} V\left(r, I_{j}\right) \leqslant \sum_{j} \int_{I_{j}}|\nabla r| d x<\varepsilon .
$$

Учитывая доказанную таким образом абсолютную непрерьвность вариации $V$ и ограниченность ее на $B$, на основании теоремы 32 из [1] (см. также [2, с. 107-110]) имеем

$$
V(r, I)=\int_{I}|\nabla r(x)| d x
$$

т.е. на самом деле в $(*)$ мы имеем равенство.

Из леммы следует

Теорема 1. Пусть множество $M \subset G$ измеримо по Лебегу и функиия $r \in$ $\mathrm{ACL}_{1}(G)$. Тогда

$$
\int_{M}|\nabla r(x)| d x=\int_{r(G)} H(\underset{t}{M}) d t
$$

ДоКАЗАТЕЛЬСТВо. Пусть $\mathscr{K}$ - кольцо подмножеств множества $G$, которые являются объединениями конечного числа полуоткрытых брусов. В силу известных свойств хаусдорфовой меры $H$ (см. [6, с. 70-86], или [7, с. 111-112]) функция

$$
\mu(M) \stackrel{\text { def }}{=} \int_{r(G)} H(\underset{t}{M}) d t
$$

есть мера (в смысле $[8, \S 7])$ на $\sigma$-алгебре $S(\mathscr{K})$ борелевских подмножеств $M \subset G$, порожденной кольцом $\mathscr{K}$. Но $\mu$ совпадает на $\mathscr{K}$ с мерой

$$
\lambda(M)=\int_{M}|\nabla r| d x
$$

поскольку из (1) следует совпадение $\mu$ и $\lambda$ на полуоткрытых брусах, входяших в $G$. Так как мера $\lambda$ является $\sigma$-конечной, она совпадает с $\mu$ на $S(\mathscr{K})($ см. $[8, \S 13])$. Но всякое измеримое по Лебегу множество $M \subset G$ представимо в виде $M=M_{0} \cup A, A \subset A_{0}$, где $M_{0}, A_{0} \in S(\mathscr{K})$ и $\operatorname{mes}_{n} A_{0}=0([8, \S 13])$. Поэтому равенство (2) распространяется на все лебеговские подмножества области $G$. 
ТЕОРема 2. Пусть функиия Ф неотрицательна и измерима по Лебегу в области $G$, а функиия $r \in \mathrm{ACL}_{1}(G)$. Тогда

$$
\int_{G} \Phi(x)|\nabla r(x)| d x=\int_{r(G)} d t \int_{G} \Phi(x) d H^{x}
$$

где интегрирование по $\underset{t}{G}$ понимается как интегрирование функиии $\Phi(x)$, определенной на пространстве $\underset{t}{G}$ с мерой $H$.

ДокАЗАТЕЛЬСтво. Пусть сначала $\Phi(x)$ - простая функция, т.е. сушествуют $l$ взаимно непересекающихся измеримых множеств $M_{1}, \ldots, M_{l}$ и $l$ действительных чисел $\alpha_{1}, \ldots, \alpha_{l}$ таких, что

$$
\Phi(x)=\sum_{k=1}^{l} \alpha_{k} \chi_{M_{k}}(x)
$$

где $\chi_{M_{k}}$ - характеристическая функция множества $M_{k} \subset G$. Тогда на $\underset{t}{G}$

$$
\Phi(x)=\Phi \cdot \chi_{t}(x)=\sum_{k=1}^{l} \alpha_{k} \chi_{M_{k}}(x)
$$

Следовательно,

$$
\int_{G} \Phi|\nabla r| d x=\sum_{k=1}^{l} \alpha_{k} \int_{M_{k}}|\nabla r| d x=\int_{r(G)} \sum_{k=1}^{l} \alpha_{k} \int_{\underbrace{G}_{t}} \chi_{M_{k}} d H^{x} d t=\int_{r(G)} d t \int_{\substack{G \\ t}} \Phi d H^{x}
$$

Если теперь $\Phi(x)$ - произвольная неотрицательная измеримая функция, существует, как известно, возрастающая последовательность $\left(\Phi_{i}\right)_{i=1,2, \ldots}$ неотрицательных простых функций, сходящаяся к $\Phi$ в области $G,[8$, с. 88]. Согласно известной теореме Леви-Лебега об интегрировании монотонно возрастающих последовательностей неотрицательных измеримых функций $[8, \S 27]$

$$
\int_{G} \Phi|\nabla r| d x=\lim _{i} \int_{G} \Phi_{i}|\nabla r| d x=\lim _{i} \int_{r(G)} d t \int_{G} \Phi_{i} d H^{x}
$$

Доказательство теоремы 2 теперь заканчивается применением указанной теоремы Леви-Лебега сначала к функциям

$$
\mathscr{F}_{i}(t)=\int_{G_{t}} \Phi_{i}(x) d H^{x}
$$

а потом к функциям $\Phi_{i}$. 
СлЕДСТВИЕ 1. Пусть $r \in \mathrm{ACL}_{1}(G)$ и пусть $\Phi(x)$ определена в области $G$, измерима по Борелю и зависит только от $r(x)$, т.е. $\Phi(x)=f \circ r(x)$. Для того, чтобь функиия $\Phi \cdot|\nabla r|$ была суммируемой на $G$, необходимо и достаточно, чтобы функиия $f(t) \cdot H(\underset{t}{G})$ была суммируемой на $r(G)$. При этом

$$
\int_{G} \Phi|\nabla r| d x=\int_{r(G)} f(t) H \underset{t}{(G)} d t .
$$

ДокАЗАтЕльство. Действительно, если $f(t) \cdot H \underset{t}{G})$ суммируема на $r(G)$, то $|f(t)| \times$ $H(\underset{t}{G})$ также суммируемая на $r(G)$ функция. В силу теоремы 2

$$
\int_{G}|\Phi(x)| \cdot|\nabla r(x)| d x=\int_{r(G)}|f(t)| \cdot H(\underset{t}{G}) d t
$$

откуда и следует суммируемость $\Phi|\nabla r|$ на $G$. Аналогично доказьвается обратное утверждение.

ЗАмЕчАнИЕ. Формула (4) является обобщением известной формулы, сводящей кратные интегралы, в которых фигурируют только функции от радиуса $t$ (определенные в $\left.\mathbb{R}^{n}\right)$, к однократным интегралам (см. $\left[9\right.$, с. 40]). В этом последнем случае $G=\mathbb{R}^{n}, G_{t}^{-}$ сфера радиуса $t$ и

$$
H(\underset{t}{G})=\frac{\pi^{n / 2}}{\Gamma(n / 2+1)} n t^{n-1}
$$

в знаменателе стоит гамма-функция Эйлера.

\section{СПИСОК ЦИТИРОВАННОЙ ЛИТЕРАТУРЫ}

[1] Кронрод А. С. О функциях двух переменных // УМН. 1950. Т. 5. №1. С. 24-134.

[2] Витушкин А. Г. О многомерных вариациях. М.: ГИТТЛ, 1955.

[3] Di Giorgi E. Su una teoria generale della misura $(r-1)$-dimensionale in un spazio ad $r$ dimensioni // Ann. Math. Pura Appl. Der. (4). 1954. V. 36. P. 191-213.

[4] Federer H. Curvature measure // Trans. Amer. Math. Soc. 1959. V. 93. № 3. P. 418-491.

[5] Fleming W. H., Rishel R. An integral formula for total gradient variation // Arch. Math. 1960. V. 11. № 3. P. 218-222.

[6] Сакс С. Теория интеграла. М.: ИЛ, 1949.

[7] Буземан Г. Выпуклые поверхности. М.: Наука, 1964.

[8] Халмош П. Теория меры. М.: ИЛ, 1953.

[9] Шварц Л. Математические методы для физических наук. М.: Мир, 1965.

Томский государственный университет

Поступило

E-mail: dean@ctc.tsu.ru

24.06 .2003 